\title{
Nonlinear optical waveguides generated in lithium niobate by swift-ion irradiation at ultralow fluences
}

\author{
J. Olivares, ${ }^{1, *}$ A. García-Navarro, ${ }^{2}$ G. García, ${ }^{2}$ A. Méndez, ${ }^{2}$ F. Agulló-López, ${ }^{2}$ A. García-Cabañes, ${ }^{3}$ \\ M. Carrascosa, ${ }^{3}$ and O. Caballero ${ }^{3}$ \\ ${ }^{1}$ Instituto de Óptica “Daza de Valdés," CSIC, C/Serrano 121, 28006-Madrid, Spain \\ ${ }^{2}$ Centro de Microanálisis de Materiales (CMAM), Universidad Autónoma de Madrid, 28049-Madrid, Spain \\ ${ }^{3}$ Departamento de Física de Materiales C-IV, Universidad Autónoma de Madrid, 28049-Madrid, Spain \\ ${ }^{*}$ Corresponding author: j.olivares@io.cfmac.csic.es
}

Received May 4, 2007; revised July 17, 2007; accepted July 28, 2007;

posted July 31, 2007 (Doc. ID 82316); published August 23, 2007

\begin{abstract}
A novel method to produce optical waveguides is demonstrated for lithium niobate $\left(\mathrm{LiNbO}_{3}\right)$. It is based on electronic excitation damage by swift ions, i.e., with energies at approximately $1 \mathrm{MeV} / \mathrm{amu}$ or above. The new technique uses high-energy medium-mass ions, such as $\mathrm{Cl}$, with electronic stopping powers above the threshold value for amorphization $(5-6 \mathrm{keV} / \mathrm{nm})$, reaching the maximum value a few micrometers inside the crystal. At the ultralow fluence regime $\left(10^{12}-10^{13} \mathrm{~cm}^{-2}\right)$ an effective nanostructured medium is obtained that behaves as an optical waveguide where light propagates transversally to the amorphous nanotracks created by every single impact. The method implies a reduction of 4 orders of magnitude with respect to He implantation. The optical waveguides present reasonable losses $(\sim 10 \mathrm{~dB} / \mathrm{cm})$ and significant secondharmonic generation (SHG) and electro-optic (EO) responses ( $>50 \%$ bulk) for the lowest fluences. (C) 2007 Optical Society of America
\end{abstract}

OCIS codes: $130.3730,130.4310,160.3730,190.4390$

Ion implantation [1] of light ions $(\mathrm{H}, \mathrm{He})$ at energies of $1-3 \mathrm{MeV}$ is a well-known procedure to fabricate optical waveguides and integrated optics devices on dielectric crystals [2]. The physical basis is the disorder and partial or full amorphization of the crystal at the end of the ion range caused by nuclear collisions between the incoming ions and the atoms of the material. A main disadvantage is that the required ion fluence (and therefore irradiation time) to achieve amorphization is very high $\left(10^{16}-10^{17} \mathrm{~cm}^{-2}\right)$. This feature has hindered the routine commercial use of ion implantation, so cheaper and simpler methods such as metal in-diffusion [3,4] or proton-exchange $[5,6]$ are mostly used for $\mathrm{LiNbO}_{3}$. This crystal has been used in the present work since it is still a reference material in photonics for electro-optic (EO) and nonlinear optical applications [7]. Recently, the use of heavier ions and higher energies to induce damage and amorphization via electronic excitations has started to be explored. A method to produce high index-jump optical waveguides producing thick buried amorphous layers by accumulation of (subthreshold) electronic damage with moderate fluences $\left(\sim 10^{14} \mathrm{~cm}^{-2}\right)$ was demonstrated in $\mathrm{LiNbO}_{3}$ [8] and KGW [9] and is an alternative to light ion implantation.

The purpose of this Letter is to go a step further and report on a novel method to fabricate a nanostructured effective medium for $\mathrm{LiNbO}_{3}$ that acts as an optical waveguide. It dramatically reduces the required ion fluences down to $\sim 10^{12} \mathrm{~cm}^{-2}$, while essentially keeping the nonlinear optical performance. The physical basis is as follows: Irradiation with medium- mass ions having sufficiently high energy so that their electronic stopping power $\left(S_{e}\right)$ is above a certain threshold value $\left(S_{t h} \approx 5-6 \mathrm{keV} / \mathrm{nm}\right.$ for $\left.\mathrm{LiNbO}_{3}\right)$ generates amorphous tracks with a nanometric diameter [10-13]. Each track is associated with a single ion impact and presents $[12,13]$ an isotropic refractive in$\operatorname{dex}\left(n_{a}=2.10\right.$ at $\left.\lambda=633 \mathrm{~nm}\right)$. It has been shown [14] that for $\mathrm{LiNbO}_{3}$ and many other oxides the radius $R_{c}(z)$ of the track at a depth $z$ increases monotonically with the stopping power $S_{e}(z)>S_{t h}$. If the irradiation parameters are chosen in such a way that $S_{e}$ increases with depth up to a certain maximum and then decreases, one expects tracks [13] whose crosssectional area has the profile illustrated in Fig. 1(a) as a function of depth. This is the situation for irradiations with $\mathrm{Cl}$ at $46 \mathrm{MeV}$, in accordance with the stopping power curves shown in Fig. 1(b). For multiple random ion impacts, the optical response of the irradiated material can be derived from an effective field approach for the dielectric permittivity, and so the effective refractive index results from an adequate averaging of those for the crystalline $\left(n_{o}\right.$ $=2.287$ and $n_{e}=2.203$ at $\left.\lambda=633 \mathrm{~nm}\right)$ and amorphous $\left(n_{a}=2.10\right)$ regions. It is clear that the outside surface layer would have a decreasing refractive index profile and would cause light waveguiding [illustrated in Fig. 1(a)] with the intensity profile of the fundamental mode. The remarkable feature is that the optical waveguides are expected to be produced at substantially lower fluences than in the standard implantation procedure. In fact, the fluences used in our work, $10^{11}-10^{13} \mathrm{~cm}^{-2}$, are well below those causing overlapping of the tracks $\left(\sim 10^{13} \mathrm{~cm}^{-2}\right)$. Moreover, the index 

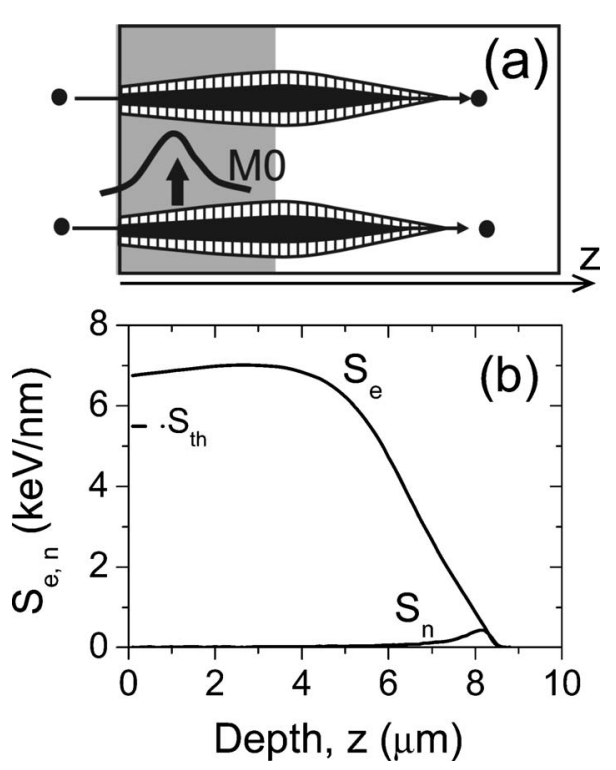

Fig. 1. (a) Schematic depth morphology of the tracks, showing the amorphous core (black) and the surrounding damage halo (dashed) generated after the passage of the ion, which stops approximately where $S_{n}$ is maximum. A schematic light profile (M0) illustrates waveguiding behavior that is generated in the top effective medium layer (emphasized with gray color). (b) Electronic, $S_{e}$, and nuclear, $S_{n}$, stopping power curves for $\mathrm{Cl} 45.8 \mathrm{MeV}$ in $\mathrm{LiNbO}_{3}$ calculated with SRIM2003. The dotted curve shows the corresponding amorphization threshold, $S_{t h}$.

profile and waveguide perfomance can be easily adjusted by suitable control of the ion energy and irradiation fluence.

The irradiations using $\mathrm{Cl}$ at $46 \mathrm{MeV}$ were performed in the $5 \mathrm{MV}$ tandem accelerator installed at the Centro de Microanálisis de Materiales, UAM [15]. The typical beam current was $\sim 1 \mathrm{nA}$, avoiding significant charging and heating of the target. Samples were $x$-cut $\mathrm{LiNbO}_{3}$ plates purchased from PHOTOX. The effective indices of the propagating modes were determined at $\lambda=630 \mathrm{~nm}$ by the dark-mode technique using light coupling through a rutile prism. The refractive index profiles were determined from the effective indices by using the WKB method valid for smooth profiles. Losses were determined through the scattered light profile measured with a CCD camera on a $2 \mathrm{~cm}$ long sample (also used for the EO measurements). The second-harmonic generation (SHG) susceptibility coefficient $d_{33}$ relative to the bulk crystal was determined for several irradiation fluences on $x$-cut waveguides, using the emission of a doubled Nd:YAG laser $(\lambda=532 \mathrm{~nm})$ as a fundamental beam [16] in the reflection geometry. On the other hand, the absolute value of the $r_{33}$ EO coefficient was measured for TE polarization at $\lambda=633 \mathrm{~nm}$ on a sample irradiated with $\mathrm{Cl}(46 \mathrm{MeV})$ at a fluence of 2 $\times 10^{12} \mathrm{~cm}^{-2}$. The method uses a Mach-Zehnder interferometric configuration [17]. The modulating voltage, of an amplitude of 20 to $100 \mathrm{~V}$ and a frequency $\Omega=1.13 \mathrm{kHz}$, was applied parallel to the $z$ direction by two deposited $\mathrm{Al}$ electrodes separated $1 \mathrm{~mm}$ along the light-beam path inside the waveguide $(y$ direction). The $r_{33}$ EO coefficient was derived from the ra- tio between the components of the output light intensity oscillating at $\Omega$ and $2 \Omega\left(\Delta I_{\Omega}\right.$ and $\Delta I_{2 \Omega}$, respectively).

Both TE and TM modes can be measured at $\lambda$ $=633 \mathrm{~nm}$ in an $x$-cut sample after the $\mathrm{Cl}$ irradiation at fluences above $10^{12} \mathrm{~cm}^{-2}$. Figure 2 shows as an illustration the effective ordinary and extraordinary refractive indices for the observed modes at 8 $\times 10^{12} \mathrm{~cm}^{-2}$ and $2 \times 10^{12} \mathrm{~cm}^{-2}$ and the fitted index profiles. The number of observed modes decreases with fluence and below $5 \times 10^{11} \mathrm{~cm}^{-2}$ no modes propagate at the used wavelength. The refractive index profiles for such low fluences can be adequately obtained by assuming linearity with fluence. For fluences at approximately $10^{13} \mathrm{~cm}^{-2}$ or above, a full amorphous layer is generated and waveguiding at the surface disappears.

Just after irradiation, losses appear to be very high and cannot be measured with the scattering method. Therefore, post-irradiation annealing treatments to reduce losses without impairing the refractive index profiles have to be applied to the samples. A good compromise is a treatment at $250^{\circ} \mathrm{C}$ for $\sim 1 \mathrm{~h}$. Profiles, shown in Fig. 2 for the representative fluence $8 \times 10^{12} \mathrm{~cm}^{-2}$, are somewhat modified most likely due to partial track recrystallization but still allow for substantial light confinement. After such annealing, losses are reduced to $10 \mathrm{~dB} / \mathrm{cm}$, which should still be improved for some technological applications. The reasons for these high losses are not yet clear. They might be associated with residual absorption or scattering by surface roughness, which is relevant at low fluences.

The SHG coefficients $d_{33}$, relative to the bulk substrate value, are shown in Fig. 3 as a function of the irradiation fluence. As for the refractive index and due to the random distribution of the tracks, one would expect an averaging of the efficiencies corre-

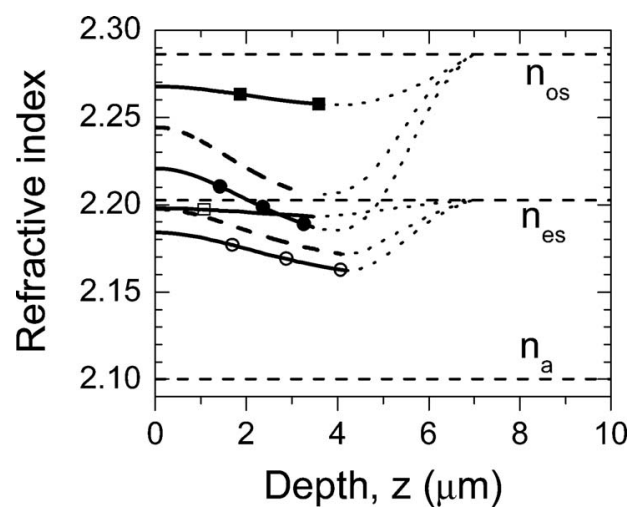

Fig. 2. Ordinary (closed symbols) and extraordinary (open symbols) refractive index profiles at $\lambda=633 \mathrm{~nm}$ for $x$-cut $\mathrm{LiNbO}_{3}$ samples irradiated with $\mathrm{Cl} 45.8 \mathrm{MeV}$ ions at the fluence $\left(\mathrm{at} / \mathrm{cm}^{2}\right)$ of $2 \times 10^{12}$ (squares) and $8 \times 10^{12}$ (circles). The symbols correspond to the measured effective indices. Horizontal dashed curves show the refractive index values for the crystal ( $n_{\mathrm{os}}$ and $\left.n_{\mathrm{es}}\right)$ and amorphous $\left(n_{\mathrm{a}}\right)$ regions. A guess of the expected refractive index profiles behind the minimum is also plotted with a dotted curve. The refractive index profiles after $1 \mathrm{~h}$ annealing in air at $250^{\circ} \mathrm{C}$ are shown with dashed curves without symbols for the fluence $8 \times 10^{12} \mathrm{~cm}^{-2}$. 


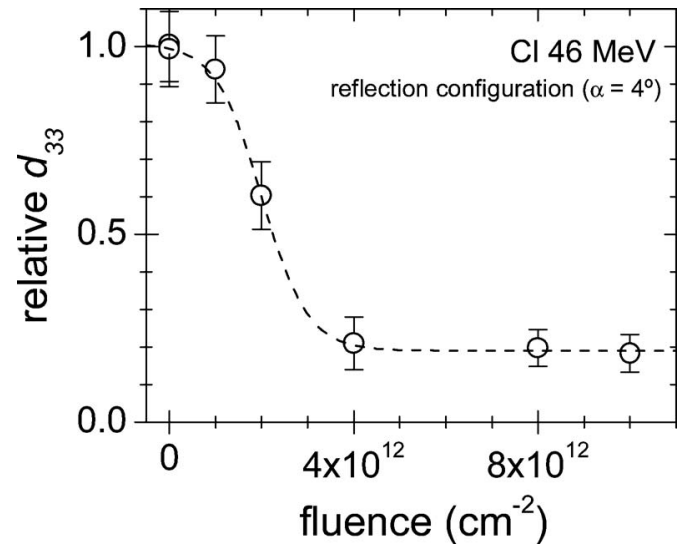

Fig. 3. Evolution of the relative $d_{33}$ nonlinear coefficient with the irradiation fluence of $\mathrm{Cl} 46 \mathrm{MeV}$ ions. The dashed curve is only a guide for the eyes.

sponding to crystalline $\left(d_{33}=25.7 \mathrm{pm} / \mathrm{V}\right.$ [18]), and amorphous $\left(d_{33}=0\right) \mathrm{LiNbO}_{3}$ regions. In fact, the data show a fast monotonic decrease as a function of fluence. For a fluence of $2 \times 10^{12} \mathrm{~cm}^{-2}$ the yield amounts to $\sim 50 \%$ of the bulk value, which is still quite significant. In accordance with the averaging model the $d_{33}$ data are approximately consistent with a radius of $\sim 2 \mathrm{~nm}$ previously measured [13] for the amorphous tracks. The consistency of the data indicates that both the linear and nonlinear optical responses of the waveguide fabricated by our new irradiation method can be well predicted.

On the other hand, the $r_{33}$ EO coefficient has been measured with good accuracy on a larger sample $(\sim 2 \mathrm{~cm}$ in length) that has been irradiated at 2 $\times 10^{12} \mathrm{~cm}^{-2}$. Figure 4 shows a clear linear dependence on the ratio $\Delta I_{2 \Omega, m} / \Delta I_{\Omega, m}$ with the modulating voltage amplitude $V_{m}$. From these data [17], a halfwave voltage, $V_{\pi}=639 \pm 16 \mathrm{~V}$, and an electro-optic coefficient, $r_{33}=13.5 \pm 0.7 \mathrm{pm} / \mathrm{V}$, have been obtained. This latter value is $\sim 40 \%$ of that for the unirradiated bulk crystal and shows a good correlation with the $d_{33}$ value (see Fig. 3).

In conclusion, novel optical waveguides have been fabricated by ultralow fluence irradiation (i.e. in the

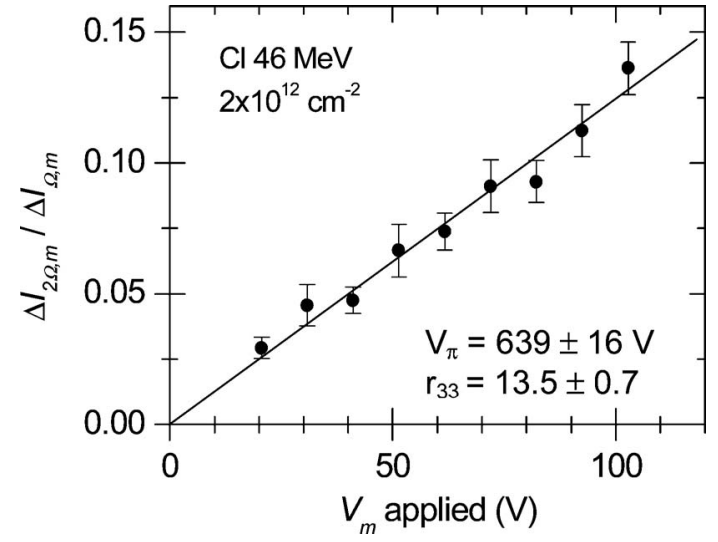

Fig. 4. Experimental dependence of the ratio $\Delta I_{2 \Omega, m} / \Delta I_{\Omega, m}$ with an amplitude of modulated voltage $V_{m}$, obtained for the electro-optic coefficient measurement. isolated track regime) on $\mathrm{LiNbO}_{3}$. The new challenging waveguides use fluences $\left(\sim 10^{12} \mathrm{~cm}^{-2}\right)$ at approximately 4 orders of magnitude below those needed in standard $\mathrm{He}$ implantation while still keeping substantial SHG yield and EO response (>50\%), although losses are relatively high at this stage. They represent a further 2 orders of magnitude reduction in fabrication time in relation to a recently proposed method [8] using fluences where strong track overlapping occurs, e.g., $\mathrm{F}(22 \mathrm{MeV})$ at $10^{14} \mathrm{~cm}^{-2}$.

We acknowledge funding from Spain's MEC project: MAT2005-06359. A. García-Navarro acknowledges the financial support of the MEC through a FPU Fellowship and of the Madrid City Hall-Residencia de Estudiantes. O. Caballero acknowledges financial support through a fellowship of the Comunidad Autónoma de Madrid.

\section{References}

1. J. F. Ziegler, Ion Implantation Technology (NorthHolland, 1992).

2. P. D. Townsend, P. J. Chandler, and L. Zhang, Optical Effects of Ion Implantation (Cambridge University Press, 1994).

3. R. V. Schmidt and I. P. Kaminow, Appl. Phys. Lett. 25, 458 (1974).

4. W. M. Young, R. S. Feigelson, M. M. Fejer, M. J. F. Digonnet, and H. J. Shaw, Opt. Lett. 16, 995 (1991).

5. M. P. de Micheli, D. B. Ostrowski, Yu. N. Korkhisko, and P. Basi, Insulating Materials for Optoelectronics: New Developments (World-Scientific, 1995).

6. J. M. Cabrera, J. Olivares, M. Carrascosa, J. Rams, R. Müller, and E. Dieguez, Adv. Phys. 45, 349 (1996).

7. Properties of Lithium Niobate, EMIS Data Review Series (INSPEC, 2002).

8. J. Olivares, G. García, A. García-Navarro, F. AgullóLópez, O. Caballero, and A. García-Cabañes, Appl. Phys. Lett. 86, 183501 (2005).

9. A. García-Navarro, J. Olivares, G. García, F. AgullóLópez, S. García-Blanco, C. Merchant, and J. Stewart Aitchison, Nucl. Instrum. Methods Phys. Res. B 249, 177 (2006)

10. B. Canut and S. M. M. Ramos, Radiat. Eff. Defects Solids 145, 1 (1998).

11. A. Meftah, J. M. Constantini, N. Khalfaoui, S. Boudjadar, J. P. Soquert, F. Studer, and M. Toulemonde, Nucl. Instrum. Methods Phys. Res. B 237, 563 (2005).

12. J. Olivares, G. García, F. Agulló-López, F. AgullóRueda, A. Kling, and J. C. Soares, Appl. Phys. A 81, 1465 (2005).

13. J. Olivares, A. García-Navarro, G. García, A. Méndez, and F. Agulló-López, Appl. Phys. Lett. 89, 071923 (2006).

14. J-H. Zollondz and A. Weidinger, Nucl. Instrum. Methods Phys. Res. B 225, 178 (2004).

15. www.uam.es/cmam

16. J. Rams and J. M. Cabrera, J. Mod. Opt. 47, 1659 (2000).

17. A. Méndez, G. De la Paliza, A. García-Cabañes, and J. M. Cabrera, Appl. Phys. B 73, 485 (2001).

18. I. Shoji, T. Kondo, A. Kitamoto, M. Shirane, and R. Ito, J. Opt. Soc. Am. B 14, 2268 (1997). 